\title{
Effectiveness of PUVA vs. UVA1 phototherapy in the treatment of morphea patients
}

\author{
Anna Malewska-Woźniak, Agnieszka Osmola-Mańkowska, Zygmunt Adamski
}

Department of Dermatology, Poznan University of Medical Sciences, Poznan, Poland

Adv Dermatol Allergol 2022; XXXIX (4): 757-761

DOI: https://doi.org/10.5114/ada.2021.108437

\begin{abstract}
Introduction: Morphea (localized scleroderma) is an inflammatory connective tissue disease, characterized by immune system dysfunction, vasculopathy and skin fibrosing. Phototherapy has been found to be effective in treating localized scleroderma. Psoralen + ultraviolet A (PUVA) and ultraviolet A1 (UVA1) phototherapy significantly enriched therapeutic possibilities.

Aim: To compare the clinical effect of PUVA photochemotherapy and UVA1 phototherapy and to evaluate the treatment response rates.

Material and methods: It was a retrospective one-centre research and observational study of all morphea patients treated with PUVA and UVA phototherapy. We reviewed phototherapy notes along with electronic and paper case records for all patients with morphea treated with PUVA and UVA1 phototherapy from January 2010 to December 2019.

Results: The study shows that patients in both groups experienced improvement based on clinical measures, resulting in a reduction in the clinical score in all groups. There is positive short- and long-term efficacy of UVA1 and PUVA phototherapy in patients with morphea. There were no statistical differences between the treatment response rates. Limitations: We had a relatively small study sample and it was a retrospective, observational study. Conclusions: Our data suggest that ultraviolet PUVA and UVA1 should be considered for the treatment of morphea with disseminated lesions or not responding to topical treatment. UVA1 is free of side effects linked to oral psoralens such as nausea, vomiting, photokeratosis, but we showed that there was no statistical advantage in the effectiveness of both. UVA1 phototherapy is, however, a less accessible form of treatment, available in the centres of higher quality.
\end{abstract}

Key words: morphea, localized scleroderma, phototherapy, UVA1, PUVA.

\section{Introduction}

Morphea, also known as localized scleroderma, is a chronic connective tissue disease. It is characterized by cutaneous sclerosis, sometimes with the involvement of underlying tissues. The aetiology of the disease is unknown despite numerous studies. It is believed that the initiating factor is trauma or infectious agents, but there is no clear evidence. Vascular changes and activation of the immune system, including autoaggressive processes are supposed to be the likely cause of morphea [1-3].

In the initial inflammatory stage of scleroderma, Th1 lymphocytes and the recently identified Th17 ones secrete pro-inflammatory cytokines, while Th2 lymphocytes dominate in the fibrotic phase [4, 5]. Morphea is clinically divided into three phases: early inflammation (active, lasting for an average of 3-4 years), progressive induration and atrophic (atrophic) [3].

According to the 2016 classification of the European Dermatology Forum, the types of morphea are as follows: limited, generalized, linear, deep and mixed [3, 6]. Morphea is characterized by rapid progression. Due to the presence of skin sclerosis, it should be differentiated from systemic sclerosis. In generalized localized scleroderma there are no changes in capillaroscopy, Raynaud's phenomenon, ulceration and resorption of phalanges, as well as facial skin involvement. These are symptoms that distinguish morphea from systemic sclerosis [3]. ANA antibodies and eosinophilia may be present in laboratory tests, which makes it difficult to correctly diagnose

Address for correspondence: Anna Malewska-Woźniak, Department of Dermatology, Poznan University of Medical Sciences, Poznan, Poland, phone: +48 608523 020, e-mail: annamalewskawozniak@gmail.com Received: 18.05.2021, accepted: 18.06.2021. 
patients and make a decision about the treatment. The treatment of localized scleroderma depends on the clinical form. There are certain factors which should be taken into account during the selection of the treatment: the severity, activity of the disease, the extent of the lesions and the age of the patient.

Most patients with reduced skin involvement in the course of morphea require topical treatment with glucocorticoids, calcineurin inhibitors, calcipotriol or phototherapy (UVA/PUVA). In more severe forms with extensive tissue involvement, systemic therapy with glucocorticosteroids and/or methotrexate, or mycophenolate mofetil should be started as soon as possible [7].

LS Skin Severity Index (LOSSI) for assessment of disease activity and severity, LS Skin Damage Index (LoSDI) for assessment of tissue damage and a general patient assessment (physician's global assessment - PGA) were proposed and validated by the Localized Scleroderma Clinical and Ultrasound Study Group. The combination of the three indices is referred to as LS Cutaneous Assessment Tool (LoSCAT) and represents a promising tool in the evaluation of patients with morphea [7]. The maximum value of each scale (mLoSSI and LoSDI) is 162 points. So far, no interpretation of the above scale has been proposed based on the score [3].

Broadband UVB, narrow-band monochromatic UVB, laser UVB, UVA in combination with psoralen (PUVA), UVA1, visible light, monochromatic light are used in dermatological phototherapy. Psoralen + ultraviolet A (PUVA) and ultraviolet A1 (UVA1) are increasingly utilized for the treatment of the patients with morphea. Phototherapy works directly on three stages of morphea lesions. It restores the balance of the immune system, reduces collagen production, and causes neovascularization [5, 7]. It should be emphasized that phototherapy has fewer side effects than methotrexate or glucocorticosteroids [8, 9]. PUVA shows the greatest efficacy in the cases of early inflammatory lesions secondary to localized scleroderma and UVA1 is better at reducing sclerotic skin lesions [7] UVA1 phototherapy $(320-400 \mathrm{~nm})$ is a commonly used treatment method for localized scleroderma [7].

\section{Aim}

The aim of this study was to compare UVA1 phototherapy and PUVA photochemotherapy and to evaluate the treatment response rates for patients with morphea. We present a retrospective review of our experience with PUVA and UVA1 phototherapy.

\section{Material and methods}

All patients with morphea treated with phototherapy (PUVA, UVA1, UVB) from January 2010 to December 2019 were included in the study group. The diagnosis of morphea was confirmed by histology examination. In all patients treated with phototherapy due to the clinical picture, limited generalized scleroderma was found. All phototherapy notes and/or paper case records for all those patients were reviewed. The data collected included age, sex, skin phototype, treatment parameters, duration of remission in weeks, the result of LS Cutaneous Assessment Tool (LOSCAT) before and 3 months after phototherapy. Patients who discontinued therapy for reasons unrelated to the therapy were excluded from analysis. We excluded (NB)UVB-treated people from the study because in this group there were only 3 patients.

\section{Ethics statement}

The retrospective study involved human participants with morphea. It was performed in accordance with the ethical standards of the local institution committee (Bioethics Commission approval number 525/18).

\section{Statistical analysis}

Statistical analysis was performed using the PQStat program version 1.6.6. Patients were divided into 3 groups, patients with morphea treated with UVA1 phototherapy, PUVA photochemotherapy and UVB phototherapy. We compared the effectiveness of treatment in two groups of patients treated with UVA1 and PUVA. We did not include the patients treated with UVB due to the small number of patients.

Interval or ordinal variables were described by descriptive statistics such as mean, median, minimum, maximum, lower and upper quartile. Nominal variables were presented as numbers and percentages. The distribution of continuous variables was verified with ShapiroWilk test. The groups were compared by Mann-Whitney test, Kruskal-Wallis test (depending on the number of compared groups) or Wilcoxon test. The Fisher exact test was used to compare categorical variables. Spearman's correlation was used to examine the associations.

A significance level of 0.05 was used for the statistical analyses carried out.

\section{Results}

The average age of all morphea patients was 50.5 years. The youngest patient in this study was 23 and the oldest was 82 years old. There were more women in the study group, which accounted for 88.6 percent of all patients, it also proves that morphea occurs more often in women than men. In all, there were 31 patients included in this trial. Thirteen of them were treated with PUVA. They had sessions three times per week and during the whole treatment they received maximum of to twenty radiotions. The remaining 18 patients received low- to medium-dose UVA1 (20-50 J/ $\left.\mathrm{cm}^{2}\right)$ phototherapy in 20-40 sessions (Table 1). The treatment was delivered five times per week. The mean age of the onset of the disease was 
Table 1. With Fisher's exact test we proved that there is no statistically significant relationship between gender and the group. Thirty-one people were qualified for our retrospective study

\begin{tabular}{lcccc}
\hline Gender & \multicolumn{2}{c}{ UVA1 } & \multicolumn{2}{c}{ PUVA } \\
\cline { 2 - 5 } & $n$ & $\%$ & $n$ & $\%$ \\
\hline Women & 15 & 16.7 & 13 & 0 \\
\hline Men & 3 & 83.3 & 0 & 100 \\
\hline All & 18 & 100 & 13 & 100 \\
\hline
\end{tabular}

42.1 years for all groups and the average duration of the disease was 18.4 months. In all patients who completed the therapy, the results obtained were satisfactory for the patients. Skin status significantly improved in all the patients who finished the treatment protocol, resulting in a significant reduction in the clinical score and also severe side effects were not observed, but a mild tanning was seen in all patients after phototherapy.

Significant reductions in LoSCAT scores were observed using both UVA1 and PUVA therapy (Figures 1, 2).

There is a statistically significant difference in the LoSCAT assessment between the two studies. The LoSCAT score was significantly reduced after phototherapy, after 3 months of treatment with both methods of phototherapy.

There are no statistically significant differences in the effectiveness of treatment in both men and women ( $p=$ 0.9379).

There are no statistically significant differences in the effectiveness between different phototypes (Table 2). The phototype was found to have no effect on the efficacy of therapy in patients with morphea. On the other hand, there was a monitoring relationship between the age and the effectiveness of treatment ( $p=0.0062$ ). The age of the patients affects the effectiveness of treatment of (patients with) morphea. The older the age, the more effective the treatment was, which resulted in a greater difference in the LoSCAT scale. There is a statistically significant monotonic relationship between the disease onset and the efficacy of the therapy $(p=0.0072)$. The later the onset of the disease, the greater the difference in the LOSCAT score, which gives greater treatment satisfaction in patients with morphea.

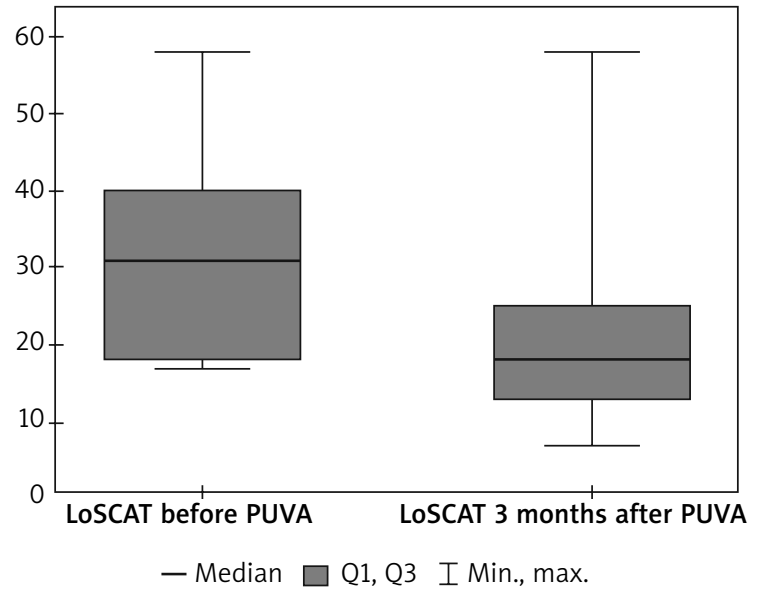

Figure 1. There is a statistically significant difference in LoSCAT assessment in the group where patients were treated with PUVA therapy. The LoSCAT score before the therapy was significantly higher than after 3 months $(p=0.0038)$ (Wilcoxon test)

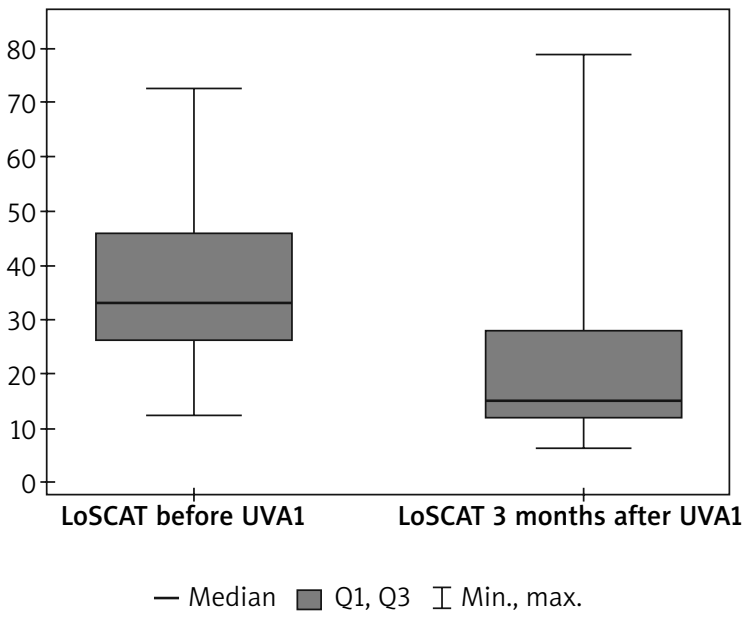

Figure 2. There was a statistically significant difference in LoSCAT scores. The LOSCAT score before UVA1 treatment was significantly higher than after 3 months $(p=0.0004)$ (Wilcoxon pair order test)

Table 2. There are no statistically significant differences in the clinical response of patients treated with phototherapy between different phototypes. $N$ either phototype has an advantage over the other in response to treatment (KruskalWallis test)

\begin{tabular}{lcccccccc}
\hline & Phototype & $N$ & Median & Minimum & Maximum & $\begin{array}{c}\text { Lower } \\
\text { quartile }\end{array}$ & $\begin{array}{c}\text { Upper } \\
\text { quartile }\end{array}$ \\
\hline $\begin{array}{l}\text { Clinical response } \\
\text { to phototherapy }\end{array}$ & 1 & 3 & -12 & -18 & -12 & -18 & -12 & 0.3852 \\
\cline { 2 - 26 } & 2 & 18 & -16 & -47 & 0 & -21 & -10 & -4 \\
\hline
\end{tabular}


The risk of disease recurrence after the end of both UVA1 and PUVA phototherapy does not correlate with the patient's gender and skin phototype.

The mean time of remission in the group of patients treated with PUVA phototherapy was 132.6 weeks, while in the case of the UVA1 it was 147.3 weeks until the last follow-up visit. There were no statistical differences in both groups ( $p=0.8714)$.

Our study included 2 patients who did not achieve a significant improvement in their dermatological condition after PUVA therapy, hence they were re-qualified for the treatment with the UVA1 method. In one of them, a significant improvement in the dermatological condition was visible, while in the other, the effects of PUVA therapy and UVA1 therapy were comparably unsatisfactory, therefore, treatment with methotrexate was applied and we observed good clinical response and treatment tolerance.

We observed hyperpigmentation of plaques induced by UVA1 phototherapy especially in those patients who had sclerotic and already atrophic plaques, which is the only side effect that cannot be avoided. We did not observe any long-term adverse effects of UVA1 and PUVA therapy such as photocarcinogenesis.

\section{Discussion}

Phototherapy for morphea was first reported in 1994 with the success of PUVA therapy used by Kerscher. Soon afterwards the benefits of UVA1 therapy were demonstrated in patients with morphea. Then, the researchers compared the effectiveness of different doses of UVA1, while in 2006 the most valuable was a randomized comparative study, where 64 patients were treated successively with low and moderate doses of UVA1 and a narrow UVB band. The study showed the superiority of UVA1 over UVB in clinical evaluation [10]. So far, no study comparing UVA1 and PUVA therapy has been conducted. In our study, 18 patients received low- to medium-dose UVA1 $\left(20-50 \mathrm{~J} / \mathrm{cm}^{2}\right)$ phototherapy in 20-40 sessions. The treatment was delivered five times per week. Thirteen of all patients who were treated with PUVA had sessions three times per week, with the radiation up to twenty. Clinical improvement was found to be significant in both UVA1 and PUVA-treated patients using LoSCAT evaluation. However, it seems that due to the availability, the UVA1 was chosen more often. Patients also had contraindications for psoralen. However, we have not proved the advantage of one method over the other.

PUVA is also exceptionally effective in treating patients with psoriasis and it is superior to other types of phototherapy. The indication for the treatment of PUVA is also mycosis fungoides and lymphomatous papulosis, where good efficacy is proven $[9,11]$.

The main indications for UVA1 phototherapy are atopic dermatitis and sclerosing skin disorders. As to sclerosing disorders, UVA1 phototherapy has been shown to be effective also in the treatment of acrosclerosis in the course of sys- temic sclerosis, extragenital lichen sclerosus et atrophicus, and scleroderma-like graft-versus-host disease [9, 11].

We agree that phototherapy is an attractive option for patients with morphea due to its efficacy. It is also an alternative to systemic immunosuppressives (glucocorticosteroids and methotrexate) for patients who cannot tolerate or have contraindications for these medications. However, although both methods of phototherapy seem to be fantastic treatments, they are still difficult to access. Based on the guidelines for the management of morphea, where phototherapy is the first-line treatment, such patients should be quickly referred to reference centres so that the introduced treatment could prevent the consequences leading to extensive sclerosis and limitation of mobility, especially in the limbs.

\section{Conclusions}

Morphea is associated with cosmetic and functional consequences, and the involvement of internal organs is very rare. Early diagnosis and treatment are very important to minimize damage such as a limited range of motion and contractures, which can often lead to a significant reduction in the quality of life. Morphea is an inflammatory, sclerotic skin disease that can affect the underlying soft tissues. Although the cause of morphea remains poorly understood, attention is focused on genetic predisposition, immune dysregulation, and environmental factors [12]. The main indication for UVA1 phototherapy are sclerosing skin disorders, especially morphea. PUVA therapy also has excellent effects in morphea patients.

The efficacy of phototherapy is based on three mechanisms. UV radiation induces apoptosis and necrosis. This affects inflammatory infiltration and keratinocytes. There is also modulation of the immune response. PUVA therapy increases IL-2 and INF- $\gamma$, key Th1 cytokines, and UVA therapy modulates collagen metabolism, due to the deep penetration $[11,13]$. UVA 1 phototherapy, according to data, induces better response to treatment in the patients with active and inflammatory morphea than in patients with sclerotic or atrophic plaques [14, 15].

Our study showed a positive efficacy of UVA1 and PUVA phototherapy in patients with morphea. We suggest that ultraviolet UVA1 and PUVA should be considered for the main treatment of morphea. It has to be emphasised that UVA1 is free of side effects seen with psoralen such as nausea, vomiting, but we showed that there is no statistical advantage in the effectiveness of both [15]. UVA1 and PUVA phototherapy seem to offer a very promising approach to the treatment of morphea, however the number of studies so far has been low, perhaps due to the rarity of the disease and sometimes difficult access to specialized centres capable of carrying out phototherapy.

\section{Conflict of interest}

The authors declare no conflict of interest. 


\section{References}

1. Fett N, Werth VP. Update on morphea. Part I. Epidemiology, clinical presentation, and pathogenesis. J Am Acad Dermatol 2011; 64: 217-28.

2. Fett N, Werth VP. Update on morphea. Part II. Outcome measures and treatment. J Am Acad Dermatol 2011; 64: 231-42.

3. Wolska-Gawron K, Krasowska D. Localized sclerodermaclassification and tools used for the evaluation of tissue damage and disease activity/severity. Dermatol Rev 2017; 104: 269-89.

4. Kurzinski K, Torok KS. Cytokine profiles in localized scleroderma and relationship to clinical features. Cytokine 2011; 55: 157-64.

5. Osmola-Mańkowska A, Teresiak-Mikołajczak E, DańczakPazdrowska A, et al. The role of dendritic cells and regulatory T cells in the pathogenesis of morphea. Centr Eur J Immunol 2015; 40: 103-8.

6. Kreuter A, Krieg T, Worm M, et al. German guidelines for the diagnosis and therapy of localized scleroderma. J Dtsch Dermatol Ges 2016; 14: 199-216.

7. Wolska-Gawron K, Michalska-Jakubus M, Krasowska D. Localized scleroderma - current treatment options. Dermatol Rev 2017; 104: 606-18.

8. Teske NM, Jacobe HT. Phototherapy for sclerosin skin conditions. Clin Dermatol 2016; 34: 614-22.

9. Placek W, Kaszub A, Lesiak A, et al. Phototherapy and photochemotherapy in dermatology. Recommendations of the Polish Dermatological Society. Dermatol Rev 2019; 106: 237-56.

10. Kreuter A, Hyun J, Stücker M, et al. A randomized controlled study of low dose UVA1, medium dose UVA1 and NB UVB phototherapy in the treatment of localized scleroderma. J Am Acad Dermatol 2006; 54: 440-7.

11. Singer S, Berneburg M. Phototherapy. J Dtsch Dermatol Ges 2018; 16: 1120-9.

12. Florez-Pollack S, Kunzler E, Jacobe Heidi T. Morphea: current concepts. Clin Dermatol 2018; 36: 475-86.

13. Furuhashi T, Torii K, Ikumi K, et al. Ultraviolet A1 phototherapy for the treatment of localized scleroderma. J Dermatol 2020; 47: 792-5.

14. Prasad S, Coias J, Chen H, Jacobe H. Utilizing UVA-1 phototherapy. Dermatol Clin 2020; 38: 79-90.

15. Keyal U, Bhatta A, Wang X. UVA1 a promising approach for scleroderma. Am J Transl Res 2017; 9: 4280-7. 